\title{
Pragmatic Opioid Use in Painful Diabetic Neuropathy
}

\author{
Yatan Pal Singh Balhara, ${ }^{1}$ Shalini Singh ${ }^{2}$ and Sanjay Kalra ${ }^{3}$
}

1. Department of Psychiatry, National Drug Dependence Treatment Center, All India Institute of Medical Sciences (AIIMS), New Delhi, India;

2. Institute of Liver and Biliary Sciences (ILBS), New Delhi, India; 3. Bharti Hospital, Karnal, India

$\mathrm{T}$ he management of painful diabetic neuropathy poses a tough clinical challenge. Although opioid analgesics are considered as second- or third-line agents in the management of moderate-to-severe neuropathic pain, prescription of opioids for this indication is higher than expected. This narrative review is a recommendation on how to ensure pragmatic use of opioids for those with painful diabetic neuropathy while avoiding complications such as opioid overdose, opioid diversion and the development of opioid-use disorder. Risk mitigation strategies at the level of the clinician include periodic assessment and documentation of clinical details, treatment history and psychosocial status. Using a multimodal approach to pain management, medication counselling, adherence monitoring programmes, evidence-based opioid dosing strategies and empowering patients to make treatment decisions are effective strategies in reducing risk associated with prolonged opioid use. At the organisational and policy level, using prescription drug monitoring programmes, carrying out periodic opioid utilisation reviews and providing training to patients and physicians on safe opioid use are useful, implementable strategies.

\section{Keywords}

Diabetes, neuropathy, opioids, analgesia, opioid abuse, opioid addiction

Disclosure: Yatan Pal Singh Balhara, Shalini Singh and Sanjay Kalra have no financial or non-financial relationships or activities to declare in relation to this article. Sanjay Kalra is a member of the journal's Editorial Board.

Review Process: Double-blind peer review.

Compliance with Ethics: This article involves a review of the literature and does not report on new clinical data, or any studies with human or animal subjects performed by any of the authors.

Authorship: All named authors meet the criteria of the International Committee of Medical Journal Editors for authorship for this manuscript, take responsibility for the integrity of the work as a whole and have given final approval for the version to be published.

Access: This article is freely accessible at

touchENDOCRINOLOGY.com @ Touch Medical Media 2020.

Received: 15 July 2019

Accepted: 16 December 2019

Published Online: 4 February 2020

Citation: European Endocrinology. 2020;16(1):21-4

Corresponding Author: Yatan Pal Singh Balhara. Department of Psychiatry, National Drug Dependence Treatment Center All India Institute of Medical Sciences (AllMS), New Delhi, India. E: ypsbalhara@gmail.com

Support: No funding was received in the publication of this article.
The prevalence of diabetes in the worldwide adult population has increased from $4.7 \%$ to $8.5 \%$ since 1980. ${ }^{1}$ Peripheral neuropathy is found in $30-90 \%$ of patients with diabetes mellitus, and $16-34 \%$ of people with diabetes suffer from painful diabetic neuropathy (PDN). ${ }^{2,3}$ South Asians are particularly prone to this complication which leads to decreased quality of life and increased healthcare costs. ${ }^{4}$ The symptomatic management of PDN with a single treatment approach remains a challenge. ${ }^{5}$ The current guidelines recommend different medications as first- and second-line agents. ${ }^{6} \mathrm{~A}$ review of recent recommendations described tricyclic agents (e.g., amitriptyline), serotonin-norepinephrine reuptake inhibitors (e.g., duloxetine), and gabapentinoids (e.g., pregabalin, gabapentin) as the first-line agents for symptomatic management of PDN. ${ }^{7}$ Opioid analgesics (e.g., morphine, methadone, tapentadol, tramadol) are recommended as second- or third-line agents and their use is recommended for management of moderate-to-severe neuropathic pain.? Despite the recommendations, use of opioids for the management of PDN commonly precedes that of other drugs. ${ }^{8}$ This is a narrative review that aims to provide an overview of the existing evidence on pragmatic use of opioids for those suffering from PDN while avoiding complications associated with opioid use such as overdose, diversion and development of opioid-use disorder.

Morphine and methadone are high-potency, full mu-opioid agonists that effectively manage moderate-to-severe pain. A meta-analysis on the efficacy of morphine for neuropathic pain management demonstrated equivocal efficacy of the drug (numbers needed to treat $=3.7$; range 2.6-6.5) with a maximum daily dose of $90-180 \mathrm{mg} /$ day. ${ }^{9}$ A meta-analysis of efficacy of methadone use for neuropathic pain management showed similar results, with the daily dose ranging between 10-80 mg/day. ${ }^{10}$ Long-term use of these potent mu-opioid agonists for chronic non-cancer pain is associated with an increased risk of opioid-use disorder, overdose events, fractures, myocardial infarctions and endocrinological harm. ${ }^{11}$ Opioid-use disorder is defined as a 'problematic pattern of opioid use leading to clinically significant impairment or distress'. ${ }^{12}$

Tramadol is a low-potency, centrally acting, weak mu-opioid agonist with aminergic activity that is used to manage PDN. ${ }^{5}$ It carries a risk of serotonin syndrome, especially when tricyclics are used concomitantly or doses higher than $400 \mathrm{mg} /$ day are consumed. ${ }^{13}$ Another risk with chronic tramadol use is that it lowers seizure threshold, even at low doses. ${ }^{14}$ Development of opioid-use disorder with use of tramadol is likely to be lower. Low-dose opioid use is defined as $\leq 40 \mathrm{mg}$ morphine or equivalent per day. ${ }^{15}$ The usual starting dose of tramadol (50-100 mg), is equivalent to $7.5-15.0 \mathrm{mg}$ of morphine. ${ }^{16}$ Thus, the risk of opioid-use disorder with tramadol is expected to be low. ${ }^{17}$ However, opioid-use disorder due to tramadol has been reported. ${ }^{18}$

Tapentadol is a relatively new, centrally acting, weak mu-opioid receptor agonist and norepinephrine and serotonin receptor antagonist that is commonly prescribed for management of PDN. ${ }^{19}$ 
Table 1: Risk factors associated with misuse of prescribed opioids

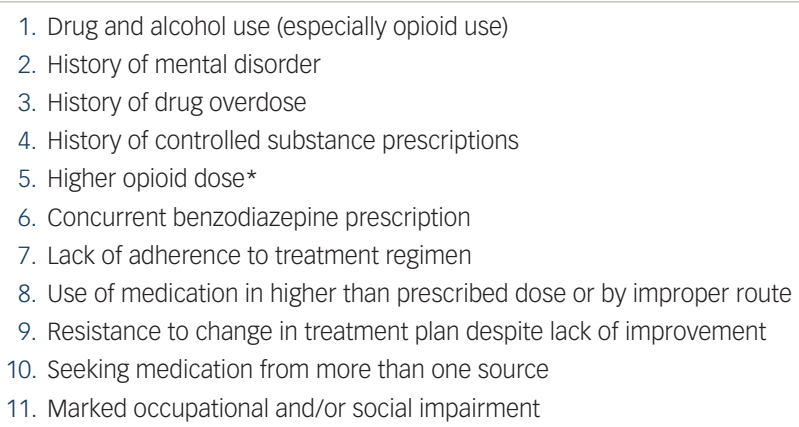

* Up to $40 \mathrm{mg}$ of morphine equivalent is considered a low dose, 41-90 mg of morphine equivalent to a moderate dose and $>90 \mathrm{mg}$ of morphine equivalent to a high dose. ${ }^{14}$

Along with pregabalin and duloxetine, it is the only drug approved by the US Food and Drug Administration for PDN management in an oral dose range of $50-700 \mathrm{mg}$ per day. ${ }^{5}$ Despite claims of low abuse potential, it has been found to be associated with opioid-use disorder in post-marketing evaluations. ${ }^{20}$

Besides the risk of development of opioid-use disorder, prolonged and/or excessive use of opioids is associated with risk of opioid overdose and other systemic complications. Hence, we offer pragmatic suggestions to ensure safe and effective use of opioid analgesics in the management of PDN. To begin with, we offer recommendations for risk mitigation that can be executed at the clinician level. In the end, we offer recommendations on risk-mitigation strategies at organisation and policy level.

\section{Risk mitigation strategies at the level of the clinician \\ Clinical review}

The first step is a thorough clinical assessment of patients with PDN to ensure that the diagnosis is correct and comprehensive. Other metabolic, toxic and inflammatory causes of neuropathic pain such as vitamin B12 deficiency or hypothyroidism and restless leg syndrome, might be contributing to pain and should be ruled out. ${ }^{2}$ Detailed clinical assessment and documentation of the presenting complaints, such as pain (quality, intensity, diurnal pattern, accompanying complaints, etc.), general medical history and past history of medical, surgical history, and psychiatric disorders, are necessary. Documentation of family history, personal history, psychosocial history (for e.g. premorbid personality) and current physical and psychological status should be done. A periodic quantitative assessment and documentation of pain severity and quality of life is an important part of clinical review.21,22

\section{Drug history review}

While considering opioid therapy for PDN, the healthcare provider should enquire about past or current history of substance-use disorder, prescription drug misuse, as well as details of past opioid prescriptions. A history of treatment non-adherence and aberrant drug use behaviours should also be taken. Table 1 enumerates the various factors that are associated with prescribed opioid misuse and could be considered as red flags when considering treatment with opioid analgesics or after having started opioids. ${ }^{14}$

\section{Treatment review}

opioid analgesics are only to be considered when multiple first-line agents (tricyclics, serotonin-norepinephrine reuptake inhibitors [SNRIS] and gabapentinoids) cannot control pain alone or in combination. ${ }^{5}$ Hence, ensuring adherence to treatment regimen using these agents is important before considering opioids. The time and dose of administration should be checked and attuned to the diurnal variation in symptoms. For example, administer duloxetine or pregabalin 1-2 hours before expected onset of symptoms. The next step is to ensure that these drugs have been used in maximally tolerated doses before using opioids as an adjunct medicine. Try interchanging drugs (from duloxetine to pregabalin, or vice versa) or augmentation strategies (from monotherapy to combination therapy). ${ }^{2}$

A multimodal, multidisciplinary approach to pain management needs to be employed during management of PDN. Non-pharmacological therapy for PDN such as physiotherapy, psychological therapies (e.g., cognitive behavioural therapy, pain-coping skills training, mindfulness-based interventions) and interventional procedures must be considered. ${ }^{11}$ Referring the patient to a pain specialist for more holistic management is a good strategy..$^{23}$

\section{Using screening tools for risk stratification of patients}

These tools, in conjunction with clinical assessment, could lead to more accurate risk prediction of outcomes such as opioid misuse and opioid-use disorder. The Opioid Risk Tool is one such validated tool. ${ }^{24}$ The revised Screener and Opioid Assessment for Patients with Pain is also used to predict subsequent aberrant drug use behaviors. ${ }^{20}$ Available literature on these screening tools is on diagnostic accuracy, but evidence on effectiveness of these tools in predicting outcomes related to opioid-use disorder is insufficient. These tools can be pre-emptively used to identify patients who would need a greater degree of clinical supervision.

\section{Medication counselling}

Effective patient education and counselling maximises patient involvement in treatment. A decision to start opioid analgesics should be the result of shared decision-making by the patient and physician. 15,11 $^{-1}$ It should be considered after exhausting other treatment options. At the same time, certain patients might be wary of starting opioids, despite inadequate pain management on other treatment. ${ }^{25}$ So a realistic cost-benefit analysis of starting opioid analgesics for PDN must be done and results discussed with patients and caregivers. Inform them about treatment goals, and possible adverse effects like constipation, nausea, vomiting, pruritis, myoclonus, drowsiness, risk of fall, endocrinological disturbances and cognitive dysfunction. ${ }^{26}$ The risk of opioid-use disorder, overdose, and need to avoid co-administration with alcohol and benzodiazepines must be clear to the patient.

\section{Pain medicine agreements and urine drug testing}

These agreements are essentially patient-clinician treatment contracts that enlist the risks and benefits to be expected from opioid use, an undertaking not to alter prescription, exceed prescribed daily dose, overshoot prescribed duration of therapy, or share one's medication supply with others. Usually, these agreements also stipulate periodic urine drug testing to detect opioid-use disorder or diversion and for adherence monitoring. ${ }^{27}$ There is fair, but limited, evidence supporting the use of pain medicine agreements and urine drug testing for high-risk patients on chronic opioid therapy. ${ }^{15}$ In light of insufficient evidence regarding their effectiveness and ethical concerns over their use, further research is needed to get more clarity on the objectives, active elements, and justifications for using these strategies. ${ }^{28}$ The agreements need to be more patient centric. ${ }^{29}$ 


\section{Drug prescription}

The PDN symptomatology may exhibit a highly variable course. Opioids should be prescribed in a minimum required dose for symptom relief. Although there are no significant differences between long- and short-acting opioids on effectiveness and adverse effect outcomes, short-acting opioids at the lowest possible dose should be preferred..$^{11,15}$ If painful symptoms exacerbate at night or in the case of breakthrough pain, short-acting preparations should be preferred for emergency dosing. ${ }^{29}$ Prescriptions should be for a finite period of time, and automatic refill should not be allowed. If a long-acting opioid is prescribed, dose titration needs to be done cautiously since the drug accumulates, leading to adverse events. Since the underlying theme of opioid-dosing recommendations is to prescribe the lowest possible dose, patients should be empowered, and encouraged, to down-titrate doses when their health permits. The use of scored tablets helps in facilitating patient-centric down-titration of doses. ${ }^{30}$ Symptomatic worsening may be mitigated by initiating or intensifying treatment with other neurotropic drugs, approved for use in PDN.

\section{Opioid dosing strategies}

There is insufficient evidence to refute or support use of scheduled continuous dosing versus as-needed dosing. Studies have shown that time-scheduled dosing is associated with higher overall opioid dosage as compared with as-needed dosing, but a review of the evidence indicates that there is no discernible difference between the two in terms of pain and quality-of-life outcomes. ${ }^{31,32}$ On the issue of opioid rotation versus maintenance of current therapy, the recommendation is that the decision to undergo opioid rotation needs to be taken in the context of low probability of a successful outcome, co-morbidities, concomitant pharmacotherapy and logistics. ${ }^{33}$ As far as the tapering protocols are concerned, the Centers for Disease Control and Prevention (CDC) recommends tapering off opioids weekly at a rate of $10-50 \%$ of the original dosage, but this should be individualised. ${ }^{34}$

\section{Using alternative drug formulations}

Tramadol, tapentadol and morphine are available as extended-release and long-acting (ER/LA) preparations. In theory, using them would help avoid the sudden spike in opioid levels, thereby reducing the abuse potential of analgesics. However, currently there is insufficient evidence to support use of ER/LA preparations to improve outcomes related to opioid-use disorder. Prescription of ER/LA preparations could be associated with an increased risk of opioid-use disorder and overdose, as per a large cohort study. ${ }^{35}$ The American Society of Interventional Pain Physicians recommends short-acting preparations at the lowest possible dose for management of chronic non-cancer pain. ${ }^{15}$

Use of deterrent or tamper-resistant formulations is a novel pharmaceutical strategy to prevent individuals from resorting to crushing or dissolving pills and then snorting or injecting them for increased bioavailability. Development of such formulations is in nascent stage and evidence supporting the benefit in mitigating opioid-use disorder is insufficient. ${ }^{36} \mathrm{~A}$ recent randomised controlled trial indicated that transdermal buprenorphine could be a useful agent in PDN, if common adverse effects such as nausea and vomiting are managed effectively. ${ }^{37}$

\section{Periodic screening and follow-up}

Patients with PDN on opioid therapy should be screened regularly for emergence of opioid-use disorder. Regular screening for psychiatric conditions such as depression and anxiety may also be indicated. validated tools to screen for opioid-use disorder are available. The
Table 2: Opioid-use disorder diagnostic criteria (based on American Psychiatric Association's Diagnostic and Statistical Manual-5) ${ }^{12}$

Opioid-use disorder is diagnosed if two or more of the following criteria are present in a person taking opioids in a 12-month period:*

- opioids are often taken in larger amounts or over a longer period than was intended;

- there is a persistent desire or unsuccessful efforts to cut down or control opioid use;

- a great deal of time is spent in activities necessary to obtain the opioid, use the opioid, or recover from its effects;

- craving, or a strong desire or urge to use opioids;

- recurrent opioid use resulting in a failure to fulfil major role obligations at work, school, or home;

- continued opioid use despite having persistent or recurrent social or interpersonal problems caused or exacerbated by the effects of opioids;

- important social, occupational or recreational activities are given up or reduced because of opioid use;

- recurrent opioid use in situations in which it is physically hazardous;

- continued opioid use despite knowledge of having a persistent or recurrent physical or psychological problem that is likely to have been caused, or exacerbated by, the substance;

- exhibits tolerance; ${ }^{*+}$ or

- exhibits opioid withdrawal. ${ }^{* \neq}$

* Patients who are prescribed opioid medications for analgesia may exhibit withdrawal and tolerance, but would not necessarily be considered to have a substance-use disorder.

Tolerance is defined as: 1) a need for markedly increased amounts of opioids to achieve intoxication or desired effect, or 2) a markedly diminished effect with continued use of the same amount of an opioid. ${ }^{12}$

¥Opioid withdrawal is defined as three (or more) of the following, developing within minutes to several days after cessation of (or reduction in) opioid use that has been heavy and prolonged (several weeks or longer), or administration of an opioid antagonist after a period of opioid use: dysphoric mood, nausea or vomiting, muscle aches, lacrimation or rhinorrhoea, pupillary dilation, piloerection, sweating, diarrhoea, yawning, fever, or insomnia. ${ }^{38}$

Current Opioid Misuse Measure is a 17-item questionnaire developed and validated to identify those patients who are currently misusing their prescribed opioid medication. ${ }^{38}$ The Addiction Behavior Checklist is a 20-item instrument which can identify problematic behaviour associated with long-term opioid use in patients with chronic pain. ${ }^{39}$ Individualised treatment that is periodically reassessed, using urine drug testing, pill counts, or other measures if needed, should form part of follow-up visits. The CDC recommends that clinicians should frequently reassess patients for pain and quality-of-life outcomes, and adverse events in the first month after initiating opioids for non-malignant pain. ${ }^{11}$ The subsequent follow-up rate could be tailored to the patient's needs and should occur within a 1-4 week range.

\section{Stakeholder involvement}

opioid-use disorder is defined as a 'problematic pattern of opioid use leading to clinically significant impairment or distress'.12 Table 2 lists the diagnostic criteria of opioid-use disorder as per the American Psychiatric Association's Diagnostic and Statistical Manual-5. ${ }^{12}$ Opioid-use disorder exists on a continuum of severity; meeting more than one of the criteria of opioid-use disorder could have serious treatment implications. All members of the diabetes care team and patients' caregivers, must be aware of the symptoms and signs of opioid-use disorder. This may help in early recognition and limitation of this disorder. An individual taking prescribed opioids under medical supervision might exhibit some of these criteria, such as tolerance and withdrawal, but that would not necessarily mean that they have opioid-use disorder. Educational and training sessions should be provided to patients and caregivers on the safe use of opioids, disposal of expired medications and safe storage of opioids. ${ }^{22}$ 
Table 3: Pragmatic suggestions to limit opioid-use disorder in persons with diabetic painful neuropathy

\begin{tabular}{|l|l|l|}
\hline Pre-prescription & With prescription & Post-prescription \\
\hline $\begin{array}{l}\text { Clinical review: } \\
\text { rule out other treatable } \\
\text { causes of pain, relevant } \\
\text { clinical details }\end{array}$ & $\begin{array}{l}\text { Medication counselling: } \\
\text { expected benefits, side } \\
\text { effects, limitations }\end{array}$ & $\begin{array}{l}\text { Adherence } \\
\text { monitoring: urine } \\
\text { drug testing, pill } \\
\text { counts, frequent } \\
\text { follow-up, risk } \\
\text { monitoring with tools }\end{array}$ \\
\hline $\begin{array}{l}\text { Pharmacological review: } \\
\text { review drug history }\end{array}$ & $\begin{array}{l}\text { Drug prescription: } \\
\text { low-dose opioids, } \\
\text { short-acting for shortest } \\
\text { possible duration }\end{array}$ & $\begin{array}{l}\text { Empower and inform } \\
\text { patient to make } \\
\text { treatment decisions }\end{array}$ \\
\hline $\begin{array}{l}\text { Treatment review: } \\
\text { record treatment rationale, } \\
\text { process and outcomes }\end{array}$ & $\begin{array}{l}\text { Plan non-pharmacological } \\
\text { management: cognitive } \\
\text { behavioural therapy, } \\
\text { pain-coping skills training, } \\
\text { mindfulness-based } \\
\text { therapy }\end{array}$ & $\begin{array}{l}\text { All-round } \\
\text { stakeholder } \\
\text { involvement for } \\
\text { timely identification } \\
\text { of problematic } \\
\text { behaviour }\end{array}$ \\
\hline $\begin{array}{l}\text { Risk stratification: assess } \\
\text { risk of opioid-use disorder }\end{array}$ & and & \\
\hline
\end{tabular}

Table 3 summarises all the recommendations discussed above to ensure pragmatic use of opioids by a physician during management of PDN.

\section{Risk mitigation strategies at organisation/ policy level}

Prescription drug monitoring programmes (PDMPs) are databases containing information on controlled drug prescriptions that can reduce problems such as duplicate prescriptions and unavailability of a patient's treatment history. ${ }^{14,40}$ Instead of using PDMPs as a means to reject a patient for treatment with opioid analgesics, current guidelines advise using PDMP data as an opportunity to discuss safety concerns regarding opioid prescriptions.11

Periodic retrospective opioid utilisation reviews by healthcare providers is an organisation-level strategy that helps promote pragmatic opioid use. ${ }^{33}$ Checking photo identification before dispensing opioid medications at pharmacies could help detect fraudulent prescriptions. ${ }^{41}$

Training of physicians is needed in two areas. First, training in pain management should be stressed upon in the undergraduate and postgraduate curriculum. ${ }^{42}$ Second, inappropriate prescribing of opioids i.e., prescribing for the wrong indication, incorrect dosage, off-label use and making conversion errors can be avoided by training physicians in safe opioid prescribing practices. ${ }^{22}$ Ensuring that permission to prescribe opioids is granted only after the necessary training in pain management, and to specialists who have reasonably demonstrated competency in management of PDN, could act as a useful deterrent against inappropriate opioid prescriptions. Since opioids are controlled drugs, physicians should be abreast of the legal status of the medications and the rules for prescribing as per local legislation.

\section{Conclusion}

The epidemic of diabetes shows no sign of abating. It may be expected, therefore, that prescription opioids for PDN among people with diabetes mellitus will continue to increase. The pragmatic suggestions shared in this narrative review should help limit the potential adverse consequences associated with prolonged opioid use among those using these medicines for PDN. The principles described here are relevant not only to PDN care, but to the management of other chronic painful conditions as well that require prescription of opioid analgesics. $\square$
1. World Health Organisation. Diabetes. 2018. Available at: www.who.int/news-room/fact-sheets/detail/diabetes (accessed 7 January 2020)

2. Callaghan $B C$, Cheng $H T$, Stables $C L$, et al. Diabetic neuropathy: clinical manifestations and current treatments. Lancet Neurol. clinical manifestation

3. Ziegler D, Rathmann W, Dickhaus T, et al, KORA Study Group. Neuropathic pain in diabetes, prediabetes and normal glucose tolerance: the MONICA/KORA Augsburg Surveys S2 and S3. Pain Med. 2009; 10:393-400.

4. Abbott CA, Malik RA, van Ross ERE, et al. Prevalence and characteristics of painful diabetic neuropathy in a large community-based diabetic population in the U.K. Diabetes Care. 2011;34:2220-4.

5. Javed S, Petropoulos IN, Alam U, Malik RA. Treatment of painful diabetic neuropathy. Ther Adv Chronic Dis. 2015;6:15-28.

6. Spallone $V$. Management of painful diabetic neuropathy: guideline guidance or jungle? Curr Diab Rep. 2012;12:403-13. Ziegler D, Fonseca V. From guideline to patient: a review of recent recommendations for pharmacotherapy of painful diabetic neuropathy I Diabetes Complicat. 2015;29:146-56.

8. diabetic neuropathy. J Dlabetes Complicat. 2015;29:146-56.
Patil PR, Wolfe J, Said Q, et al. Opioid use in the management of diabetic peripheral neuropathy (DPN) in a large commercially insured population. Clin J Pain. 2015;31:414-24.

9. Cooper TE, Chen J, Wiffen PJ, et al. Morphine for chronic neuropathic pain in adults. Cochrane Database Syst Rev. 2017;5:CD011669.

10. MCNicol ED, Ferguson MC, Schumann R. Methadone for neuropathic pain in adults. Cochrane Database Syst Rev. 2017;5:CD012499.

11. Dowell D, Haegerich TM, Chou R. CDC guideline for prescribing opioids for chronic pain--United States, 2016. JAMA. 2016;315:1624-45

12. Substance-related and addictive disorders. In: American Psychiatric Association. Diagnostic and Statistical Manual of Mental Disorders (DSM-5 $5^{\oplus}$. Washington DC, USA: American Mental Disorders (DSM-5®). Washington DC, USA:An
Psychiatric Association Publishing. 2013:481-91.

13. Spiller HA, Gorman SE, Villalobos D, et al. Prospective multicenter evaluation of tramadol exposure. J Toxicol Clin Toxicol. 1997;35:361-4.

14. Beyaz SG, Sonbahar T, Bayar F, Erdem AF. Seizures associated with low-dose tramadol for chronic pain treatment. Anesth Essays Res. 2016;10:376-8.

15. Manchikanti L, Kaye AM, Knezevic NN, et al. Responsible, safe, and effective prescription of opioids for chronic non-cancer pain: American Society of Interventional Pain Physicians (ASIPP) guidelines. Pain Physician. 2017;20:S3-92.
16. Lewis KS, Han NH. Tramadol: a new centrally acting analgesic. Am J Health Syst Pharm. 1997;54:643-52.

17. Edlund $\mathrm{MJ}$, Martin $\mathrm{BC}$, Russo $\mathrm{JE}$, et al. The role of opioid prescription in incident opioid abuse and dependence among individuals with chronic noncancer pain: the role of opioid individuals with chronic noncancer pain:
prescription. Clin 」 Pain. 2014:30:557-64.

18. Salgaonkar S. At the opioid crossroad for chronic non cancer Salgaonkar S. At the opioid cros
pain. Indian J Pain. 2018;32:57.

19. Singh DR, Nag K, Shetti AN, Krishnaveni N. Tapentado hydrochloride: a novel analgesic. Saudi J Anaesth. 2013; 7:322-6.

20. Butler SF, McNaughton EC, Black RA. Tapentadol abuse potential: a postmarketing evaluation using a sample of individuals evaluated for substance abuse treatment. Pain Med. 2015;16:119-30.

21. Smith SC, Lamping DL, Maclaine GDH. Measuring health-related quality of life in diabetic peripheral neuropathy: a systematic review. Diabetes Res Clin Pract. 2012;96:261-70.

22. Hawker GA, Mian S, Kendzerska T, French M. Measures of adult pain: Visual Analog Scale for Pain (VAS Pain), Numeric Rating Scale for Pain (NRS Pain), McGill Pain Questionnaire Rating Scale for Pain (NRS Pain), McGill Pain Questionnaire
(MPO), Short-Form MCGill Pain Questionnaire (SF-MPQ) (MPQ), Short-Form McGill Pain Questionnaire (SF-MPQ),
Chronic Pain Grade Scale (CPGS), Short Form-36 Bodily Pain Scale (SF-36 BPS), and measure of Intermittent and constant Osteoarthritis Pain (ICOAP). Arthritis Care Res (Hoboken). 2011;63(Suppl. 11):S240-52.

23. Hahn KL. Strategies to prevent opioid misuse, abuse, and diversion that may also reduce the associated costs. Am Health Drug Benefits. 2011;4:107-14.

24. Webster LR, Webster RM. Predicting aberrant behaviors in opioid-treated patients: preliminary validation of the Opioid Risk Tool. Pain Med. 2005;6:432-42.

25. Graczyk M, Borkowska A, Krajnik M. Why patients are afraid of opioid analgesics: a study on opioid perception in patients with opioid analgesics. a study on opioid perception in patic
chronic pain. Pol Arch Intern Med. 2018;128:89-97.

26. Baldini A, Von Korff M, Lin EHB. A review of potential adverse effects of long-term opioid therapy: a practitioner's guide. Prim Care Companion CNS Disord. 2012:14. PII: PCC. 11m01326.

27. Fishman SM, Bandman TB, Edwards A, Borsook D. The opioid contract in the management of chronic pain. J Pain Symptom Manage. 1999;18:27-37.

28. Arnold RM, Han PKJ, Seltzer D. Opioid contracts in chronic nonmalignant pain management: objectives and uncertainties. Am J Med. 2006;119:292-6.

29. Albrecht JS, Khokhar B, Pradel F, et al. Perceptions of patient provider agreements. J Pharm Health Serv Res. 2015;6:139-44
30. Chou R, Fanciullo GJ, Fine PG, et al. Clinical guidelines for the use of chronic opioid therapy in chronic noncancer pain. J Pain . 2009:10:113-30

31. Von Korff M, Merrill JO, Rutter $\mathrm{CM}$, et al. Time-scheduled versus pain-contingent opioid dosing in chronic opioid therapy. Pain. 2011:152:1256-62

32. Drew $D$, Gordon $D$, Renner $L$, et al. The use of "as-needed" range orders for opioid analgesics in the management of pain: a consensus statement of the American Society of Pain Management Nurses and the American Pain Society. Pain Manag Nurs. 2014;15:551-4.

33. Fine PG, Portenoy RK. Establishing "best practices" for opioid rotation: conclusions of an expert panel. J Pain Symptom Manage. 2009;38:418-25.

34. Centers for Disease Control and Prevention. CDC guideline for prescribing opioids for chronic pain. 2018. Available at: www.cdc.gov/drugoverdose/prescribing/guideline.html (accessed 7 January 2020).

35. Liu Y, Logan JE, Paulozzi $\amalg$, et al. Potential misuse and inappropriate prescription practices involving opioid analgesics. Am J Manag Care. 2013;19:648-65.

36. Lourenco LM Matthews M. Jamison RN. Abuse-deterrent and tamper-resistant opioids: how valuable are novel formulations in thwarting non-medical use? Expert Opin Drug Deliv. 2013;10:229-40

37. Simpson RW, Wlodarczyk JH. Transdermal buprenorphine relieves neuropathic pain: a randomized, double-blind, parallel-group, placebo-controlled trial in diabetic peripheral neuropathic pain. Diabetes Care. 2016;39:1493-500.

38. Butler SF, Budman SH, Fernandez KC, et al. Development and validation of the Current Opioid Misuse Measure. Pain. 2007;130:144-56

39. Wu SM, Compton P, Bolus R, et al. The addiction behaviors checklist: validation of a new clinician-based measure of inappropriate opioid use in chronic pain. J Pain Symptom Manage. 2006;32:342-51.

40. Bao Y, Pan Y, Taylor A, et al. Prescription drug monitoring programs are associated with sustained reductions in opioid prescribing by physicians. Health Aff (Millwood). 2016;35:1045-51

41. House Committee on Health, Welfare and Institutions House Bill No. 964. 2010. Available at: http://leg1.state. va.us/cgi-bin/legp504.exe?101+ful+HB964H1 (accessed 7 January 2020).

42. Gourlay D, Heit H. Universal precautions: a matter of mutual trust and responsibility. Pain Med. 2006;7:210-1. 